\title{
Correcting Active Scatterometer Data for the Effects of Rain Using Passive Radiometer Data
}

\author{
K. A. Hilburn, F. J. Wentz, D. K. Smith, and P. D. Ashcroft \\ Remote Sensing Systems, Santa Rosa, California
}

(Manuscript received 18 March 2005, in final form 15 August 2005)

\begin{abstract}
A model for the effects of rain on scatterometer data is proposed. Data from the Advanced Microwave Scanning Radiometer (AMSR) and the SeaWinds scatterometer, both on the Midori-II satellite, are used. The model includes three basic rain effects: attenuation, rain roughening of the sea surface, and volumetric backscatter. Attenuation is calculated directly from the radiometer data and beam filling is explicitly addressed. The model simultaneously solves for both the rain roughening and volumetric backscatter. Fitting the coefficients of the model requires an estimate of the radar cross section because of wind roughening, and NCEP Global Data Assimilation System (GDAS) wind vectors are used for this purpose. Both the derived rain roughening and volumetric backscatter are similar to results in published work, but the values are slightly smaller, especially for vertical polarization. Drop size distribution variability is accounted for by formulating the radar equation in terms of the parameters of the radiative transfer equation and using additional radiometric information. Explicit inclusion of vertical profile variability results in an underdetermined problem, but it is implicitly included in fitting the model to the data. The correction makes large improvements in wind speeds and modest improvements in wind directions. Wind statistics and specific examples are shown to illustrate the nature of the improvements. The correction is limited, however, by measurement mismatch issues and the nonlinear nature of the wind retrieval.
\end{abstract}

\section{Introduction}

The SeaWinds scatterometer has proven to be a very successful tool for measuring surface vector winds over the world's oceans. The original SeaWinds scatterometer has flown on the National Aeronautics and Space Administration (NASA) Quick Scatterometer (QuikSCAT) satellite since June 1999, providing highly accurate vector winds for $90 \%-95 \%$ of the ocean. The accuracy of the remaining $5 \%-10 \%$ of the winds is degraded because of rain contamination. Several scatterometer-alone rain flag algorithms have been developed and implemented, but determining which 5\%$10 \%$ of the data are rain contaminated through the use of only the scatterometer data is not a trivial issue (Mears et al. 2000). Moreover, these rain flag algorithms only serve to identify rain contamination and cannot correct it. The Midori-II mission included an Advanced Microwave Scanning Radiometer (AMSR)

Corresponding author address: Kyle Hilburn, Remote Sensing Systems, 438 First Street, Suite 200, Santa Rosa, CA 95401.

E-mail: hilburn@remss.com in addition to a SeaWinds scatterometer on the satellite platform, allowing us to explore solving this problem. In this analysis, the radiometer is used to identify raincontaminated scatterometer observations and then to correct for rain effects. The purpose of this paper is to describe how passive radiometric data can be used to remove the rain signal from active scatterometer data and to assess to what degree this is successful. We use the entire 6-month dataset of SeaWinds and AMSR data from the Midori-II mission.

A scatterometer is basically a radar but with a design and signal processing specialized for the task of measuring surface returns [for a general overview of scatterometry see Naderi et al. (1991); for an overview of SeaWinds see Lungu (2001) and Freilich (1996)]. Scatterometers operate at microwave frequencies that are in Bragg resonance with capillary waves on the ocean surface. Capillary waves are centimeter-scale waves arising from the balance between gravity and surface tension. The measured backscatter is referred to as the normalized radar cross section and is designated by $\sigma_{0}$. The radar cross section is a strongly increasing function of the surface wind speed is also modulated by the wind 
direction, scatterometer azimuth (look) angle relative to the wind, incidence angle, and polarization. This relationship is referred to as the geophysical model function (Wentz and Smith 1999). Several such models exist and are in use.

Rain affects scatterometer data in the following three ways: 1 ) rain reduces the transmission of the radar pulse through the atmosphere, 2) rain alters the roughness of the sea surface, and 3) the radar pulse is backscattered by the intervening raindrops. The basic equation for the effects of rain on scatterometer data (in linear units) is given by

$$
\sigma_{0, \text { meas }}=\tau^{2}\left(\sigma_{0, \text { wind }}+\Delta \sigma_{0, \text { rain }}\right)+\sigma_{0, \text { vbs }},
$$

where $\sigma_{0, \text { meas }}$ is the measured cross section, $\sigma_{0 \text {,wind }}$ is the cross section resulting from the wind alone, $\Delta \sigma_{0 \text {, rain }}$ is the change in surface roughness resulting from rain impact (may be positive or negative), $\tau^{2}$ is the atmospheric transmission, and $\sigma_{0, \text { vbs }}$ is the volumetric backscatter by rain.

The nonlinear nature of the wind retrieval process makes the effects of rain on the retrieved wind speeds and directions quite complicated (see section 6). Even small amounts of rain can have large effects on retrieved winds (Hoffman et al. 2004). Typically, the net effect of rain is to increase the retrieved wind speeds. However, in tropical cyclone cases in which the rain is very heavy and the true wind speed is high, the attenuation effect can dominate and lead to lower retrieved wind speeds. The effect of rain on wind direction retrievals is very complicated and the exact nature of the effect depends upon how the spatial distribution of the rain causes a differential effect on the various $\sigma_{0}$ observations within a wind vector cell. In general, the presence of rain tends to decrease the azimuthal signal and increase the horizontal-to-vertical polarization ratio. Both effects cause the retrieved wind directions to be oriented perpendicular to the satellite movement (i.e., the winds blow "cross track").

A few studies have investigated the effects of rain on scatterometer data. The earliest comprehensive study took a statistical approach (Stiles and Yueh 2002) by fitting a linear relationship to the measured $\sigma_{0}$ versus the wind-only $\sigma_{0}$ as determined by the National Centers for Environmental Prediction (NCEP) analysis. The slope and intercept coefficients were regressed against columnar rain rate for each of the two QuikSCAT beams. They found relationships analogous to the $k-R$ and $z-R$ relationships in meteorology, however the coefficients of the relationships were quite different than would typically be expected. Our work sheds light on the reasons for these discrepancies-accounting for subfootprint variability ("the beam-filling effect") in the slope term and accounting for rain roughening in the intercept term. Tournadre and Quilfen (2003) took a theoretical approach in their investigation. They focused on modeling the attenuation and volumetric backscatter terms and ignored any rain roughening. They found the distribution of rain within the scatterometer footprint to be very important. Draper and Long (2004) took an empirical approach using NCEP wind and Tropical Rainfall Measuring Mission (TRMM) precipitation radar data in a somewhat similar, but more in-depth study than that of Stiles and Yueh (2002). They employed a more complicated model and attempted to separate the volumetric backscatter from surface rain-roughening signals.

We begin this paper by describing the passive radiometer and active scatterometer systems on the MidoriII satellite in section 2. In section 3, we discuss how we estimate transmission and discuss the issue of beam filling. Section 4 gives an in-depth discussion of drop size distribution variability, vertical profile variability, the effective temperature depression, and how to estimate volumetric backscatter. The estimation of rain roughening is investigated in section 5. Statistical and caseby-case results from our corrected wind dataset are both presented in section 6 .

\section{Data}

This study uses data from the SeaWinds scatterometer and the AMSR that were both flown on the Midori-II satellite. The Midori-II satellite is in a sunsynchronous orbit with an altitude of $803 \mathrm{~km}$, a 101-min period, a 2230 ascending local equatorial crossing time, and a repeat period of 57 orbits or 4 days. An operational anomaly caused the premature end of the $M i$ dori-II mission, resulting in AMSR data available for the first 10 months of 2003, and a combined SeaWinds and AMSR dataset available from 10 April through 25 October 2003.

The SeaWinds scatterometer is a Ku-band (13.4 $\mathrm{GHz}$ ) scatterometer with a horizontal polarization beam at a nominal incidence angle of $47^{\circ}$ and a vertical polarization beam at a nominal incidence angle of $55^{\circ}$. SeaWinds on Midori-II has calibration and performance characteristics almost identical to SeaWinds on QuikSCAT. All of the results in this paper use data derived using the $\mathrm{Ku}-2001$ geophysical model function. Bourassa et al. (2003) performed the Ku-2000 model function validation, and the $\mathrm{Ku}-2001$ model function is very similar, except that speeds above $20 \mathrm{~m} \mathrm{~s}^{-1}$ are about $5 \%$ lower and there is a slightly stronger tendency in rain for the wind directions to turn toward a 


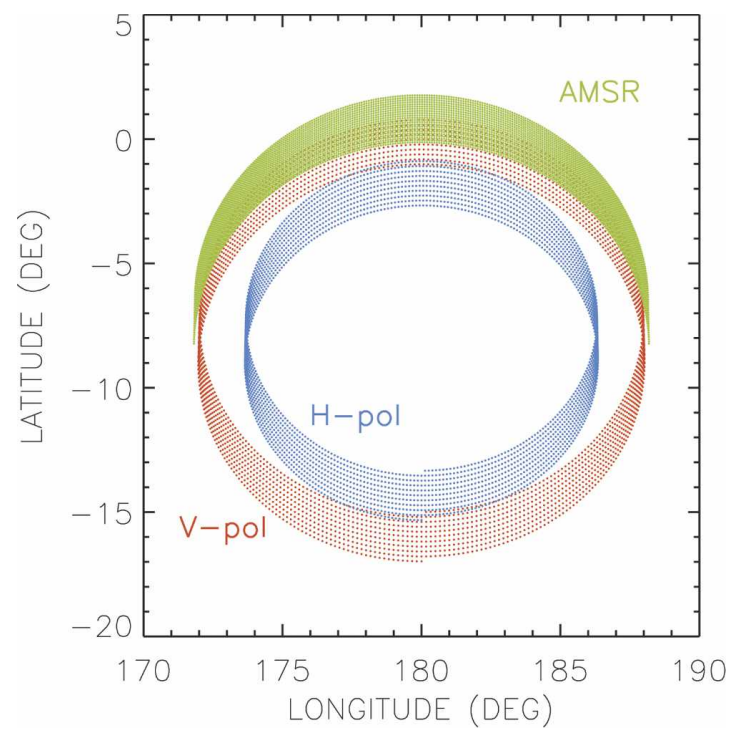

FIG. 1. Simplified simulation of AMSR (green) and SeaWinds (blue: horizontal polarization, red: vertical polarization) sampling patterns on the earth. Note that the AMSR swath is slightly wider than the SeaWinds swath. This figure depicts approximately 0.5 min of data collection.

direction that is perpendicular to the satellite track. Improvements to this model function are in development. SeaWinds on Midori-II data are available for browsing or downloading from Remote Sensing Systems (online at http://www.remss.com).

The AMSR has a large antenna (2-m diameter) that allows for high-resolution measurements (nominally 12 $\mathrm{km}$ ) from the altitude of Midori-II. The channels of direct interest in the rain retrieval are the 18.7- and 36.5-GHz channels (both vertical and horizontally polarized). AMSR has a nominal incidence angle of $55^{\circ}$.

There are four sources of mismatch between the SeaWinds and AMSR observations that limit any potential correction. While the SeaWinds and AMSR observations are nominally spatially collocated, differences in the sampling geometries cause the two instruments to view different scenes. First, SeaWinds collects data looking forward and backward, while AMSR collects data looking forward only (Fig. 1). A second difference is that the SeaWinds horizontal polarization beam has a smaller incidence angle than that of AMSR. The third difference is that the SeaWinds and AMSR footprints are different sizes. The AMSR footprint $(36.5 \mathrm{GHz})$ is nominally $12 \mathrm{~km}$, while the SeaWinds $\sigma_{0}$ eggs are nominally $25 \mathrm{~km}$. The fourth difference is that the centers of the AMSR and SeaWinds footprints are not in exactly the same locations on the earth. This is important when dealing with such an inhomogeneous field as rain. The time differences between AMSR and SeaWinds obser- vations do not exceed $2.5 \mathrm{~min}$, so this is only a small source of mismatch.

To take advantage of the existing data-processing architecture at Remote Sensing Systems, we first process the AMSR brightness temperatures ("L2A" files) to obtain geophysical parameters ("L2B" files). The processing provides the geophysical parameters at the 36.5$\mathrm{GHz}$ footprint resolution. Then, for the purpose of the rain correction, we store the SeaWinds and AMSR data in a combined swath-level data product. To create this product, we collocate the available AMSR observations whose centers fall within $25 \mathrm{~km}$ of the centers of the SeaWinds $\sigma_{0}$ observations. Typically, six AMSR observations are found, and these observations are averaged using a distance to the third power weighting in order to strongly weigh the closest observations. This is a simple, quick, and computationally inexpensive method of combining the data; however, a more complicated weighting by antenna beam pattern would be preferable.

Fitting the model (1) requires an estimate of $\sigma_{0 \text {,wind }}$. We use NCEP Global Data Assimilation System (GDAS) wind vectors for this purpose. The 10-m NCEP wind vectors are available on a $1^{\circ}$ grid 4 times per day. The vectors are interpolated in time and space to the satellite overpasses. Comparison of NCEP with buoy data binned by radiometer rain rate (not shown) has demonstrated that the quality of NCEP wind vectors do not degrade with the presence or intensity of rain and serve as a reliable estimate of the wind vector in such situations.

\section{Estimating transmission}

The first effect of rain we consider is the decrease in the transmission of the radar beam through the atmosphere because of the presence of liquid water between the sensor and the sea surface. Unlike the corrections for the volumetric backscatter and the rain roughening, which decrease the measured cross section, the correction for transmission increases the measured cross section. Because transmission is a decreasing exponential function of attenuation, the transmission correction increases quite rapidly with increasing rain rate, and if not treated correctly easily leads to winds that have been overcorrected (speeds are far too high).

Our transmission calculation accounts for the effects of oxygen and water vapor (small effects), cloud (a larger effect), and rain (the dominant effect). Calculating the transmission for oxygen and water vapor (Wentz 1997) and cloud (Wentz and Spencer 1998) attenuation is straightforward and will not be described here. Calculating the transmission in the presence of 
rain is much more complicated and deserves further elaboration.

The attenuation of microwave radiation has been well studied and is well understood (e.g., ITU Radiocommunication 1992). However, much of this study has been on ground-based communications links for which the width of the beam is small relative to the horizontal length scale of rain cells. The $25-\mathrm{km}$ scatterometer footprints are much larger than the typical $3-\mathrm{km}$ rain cell (Sauvageot et al. 1999). Thus, the horizontal inhomogeneity of the rain field cannot be ignored in our transmission calculations. For our correction, we need to obtain the footprint average value of transmission. We use the terminology "beam-filling effect" to refer to the fact that when calculating the spatial average of a nonlinear function (in this case, the exponential function), the nonlinearity acts on the inhomogeneity to produce a systematic bias.

The footprint-averaged transmission is directly estimated from dual-polarization radiometric measurements using a technique described by Petty (1994) and Wentz and Spencer (1998). The technique can be understood by considering a simplified model for the brightness temperature $T_{B}$ :

$$
T_{B}=T_{E}\left(1-\tau^{2} \rho\right),
$$

where $T_{E}$ is the effective temperature of the earthatmosphere system, $\tau^{2}$ is the two-way total transmittance through the atmosphere, and $\rho$ is the reflectivity of the sea surface. Dual-polarization measurements can then be used to separate the $\tau^{2}$ signal from the $T_{E}$ signal. The $\tau^{2}$ signal contains the attenuation information, and when rain is present, the $T_{E}$ signal contains scattering information (section $4 \mathrm{c}$ ). In (2), $\tau^{2}$ represents the footprint-averaged transmission. To be explicit, if [] is the expectation (i.e., averaging) operator, then

$$
\left[\tau^{2}\right]=\left[e^{t A}\right]=e^{t \hat{A}} \geq e^{t[A]}, \quad \hat{A} \leq[A],
$$

where $t=-2 \sec \theta, \theta$ is the incidence angle, and $A$ is the columnar attenuation. Note that $A$ is the attenuation coefficient integrated through the depth of the atmosphere. The footprint-averaged transmission is given by $\left[e^{t A}\right]$, whereas the transmission calculated from the footprint-averaged attenuation is given by $e^{t[A]}$. Note that (3) is a specific case of Jensen's inequality and the left-hand side of (3) is equivalent to the momentgenerating function of the subpixel attenuation probability distribution. We have seen (Hilburn and Wentz 2003) that typical conditions can easily make a factor of 3 difference between $\left[e^{t A}\right]$ and $e^{t[A]}$ at a rain rate of 25 $\mathrm{mm} \mathrm{h}^{-1}$. Thus, using an estimate of the footprint- averaged rain rate in order to get the average attenuation $[A]$, can easily lead to overcorrecting the cross sections by a factor of 3 . This is why we do not estimate the transmission from retrieved geophysical parameters, but instead estimate it directly from the brightness temperatures.

While the radiometer provides a direct estimate of the footprint-averaged transmission, it does not provide it at the scatterometer frequency. In a hypothetical case where a uniform rain field completely fills the footprints, the spectral relationship between the transmissions follows known theoretical values. That is to say, $\hat{A}_{36.5}=\left[A_{36.5}\right]$ and $\hat{A}_{13.4}=\left[A_{13.4}\right]$, so that $\hat{A}_{36.5} / \hat{A}_{13.4}=$ $\left[A_{36.5}\right] /\left[A_{13.4}\right]$, and the ratio $\left[A_{36.5}\right] /\left[A_{13.4}\right]$ is given by Mie theory. In the case of a nonuniform rain field, Wentz and Spencer (1998) show that not only is $\hat{A}_{36.5} \leq\left[A_{36.5}\right]$ and $\hat{A}_{13.4} \leq\left[A_{13.4}\right]$, but also $\hat{A}_{36.5} /$ $\hat{A}_{13.4} \leq\left[A_{36.5}\right] /\left[A_{13.4}\right]$. Because $\left[A_{36.5}\right]$ and $\left[A_{13.4}\right]$ represent the true footprint-averages values of attenuation, they still follow the known relationship implied by Mie theory. Thus, inferring the attenuation at one frequency from the attenuation at another frequency is made complicated by the beam-filling effect.

The radiometer provides an estimate of

$$
\tau_{36.5}^{2}=\exp \left(-2 \sec \theta \hat{A}_{36.5}\right),
$$

but we need

$$
\tau_{13.4}^{2}=\exp \left(-2 \sec \theta \hat{A}_{13.4}\right),
$$

and there is no fixed spectral relationship between $\hat{A}_{36.5}$ and $\hat{A}_{13.4}$. The ratio of the mean parameters does follow the fixed theoretical relationship implied by $\mathrm{Mie}$ theory:

$$
R_{A}=\frac{\left[A_{13.4}\right]}{\left[A_{36.5}\right]} .
$$

In the algorithm we parameterized this ratio in terms of [ $\left.A_{36.5}\right]$, and it varies from 0.12 in light absorption to 0.21 in heavy absorption. The definition of beam-filling correction factor gives

$$
\begin{aligned}
& {\left[A_{36.5}\right]=B_{36.5} \hat{A}_{36.5} \text { and }} \\
& {\left[A_{13.4}\right]=B_{13.4} \hat{A}_{13.4},}
\end{aligned}
$$

where $B_{36.5}$ and $B_{13.4}$ are the beam-filling correction factors. Combining (6a) and (6b) with (5) we get

$$
\hat{A}_{13.4}=\hat{A}_{36.5}\left(R_{A} / R_{B}\right)
$$

where

$$
R_{B}=\frac{B_{13.4}}{B_{36.5}}
$$


is the ratio of the beam-filling factors. If the variability of liquid water in the radiometer footprint is the same as the variability in the scatterometer footprint, then $R_{B}$ can be calculated analytically by assuming a form for the subpixel probability distribution function of liquid water (Wentz and Spencer 1998). In this case, $R_{B}$ goes from a value of 1 with light rain to a value of 0.4 for heavy rain. However, we have found that this produces overcorrected winds that are especially evident in tropical storms. One possible explanation for this would be if the variability of liquid water in the scatterometer footprint is larger than the variability of liquid water in the radiometer footprint. This might be reasonable given that the diameter of the scatterometer footprint is roughly twice the size of the radiometer footprint, and we would expect a larger footprint to have greater variability in it. If the variability of liquid water in the scatterometer footprint is larger than the variability in the radiometer footprint, then $R_{B}$ more-or-less assumes a constant value of a little less than one. For the purposes of our correction, we have found that a value of $R_{B}=$ 1 performs better than with smaller values. Figure 2 shows the values of transmission we use in our correction along with the values calculated assuming uniform beam filling. The primary reason for the difference between horizontal and vertical polarization in Fig. 2 is the difference in incidence angle for each SeaWinds beam. The width of the standard deviation is related to the variability of rain cloud temperature and to the variability of liquid water in the footprint for the nonuniform case. We note that for vertical polarization, our transmission values get as small as $0.4 \approx 4 \mathrm{~dB}$, and for horizontal polarization our transmission values get as small as $0.5 \approx 3 \mathrm{~dB}$. It is interesting to note that these values are very similar to those found by Stiles and Yueh (2002). The power laws that Stiles and Yueh (2002) fit to these attenuation results had coefficients quite different from those of "typical" relationships (ITU Radiocommunication 1992). The results of this section suggest the differences between their relationships and the typical relationships are due to beamfilling effects.

\section{Estimating volumetric backscatter}

The typical procedure for estimating volumetric backscatter from rain involves the use of a $z-R$ relationship, which is a power-law relationship between the radar reflectivity factor $z$ (closely related to the radar backscatter $\eta$ ) and the rain rate $R$. It is well known (e.g., Doviak and Zrnić 1993) that $z-R$ relationships vary dramatically depending upon the raindrop size distribution. The AMSR rain rates are derived from an

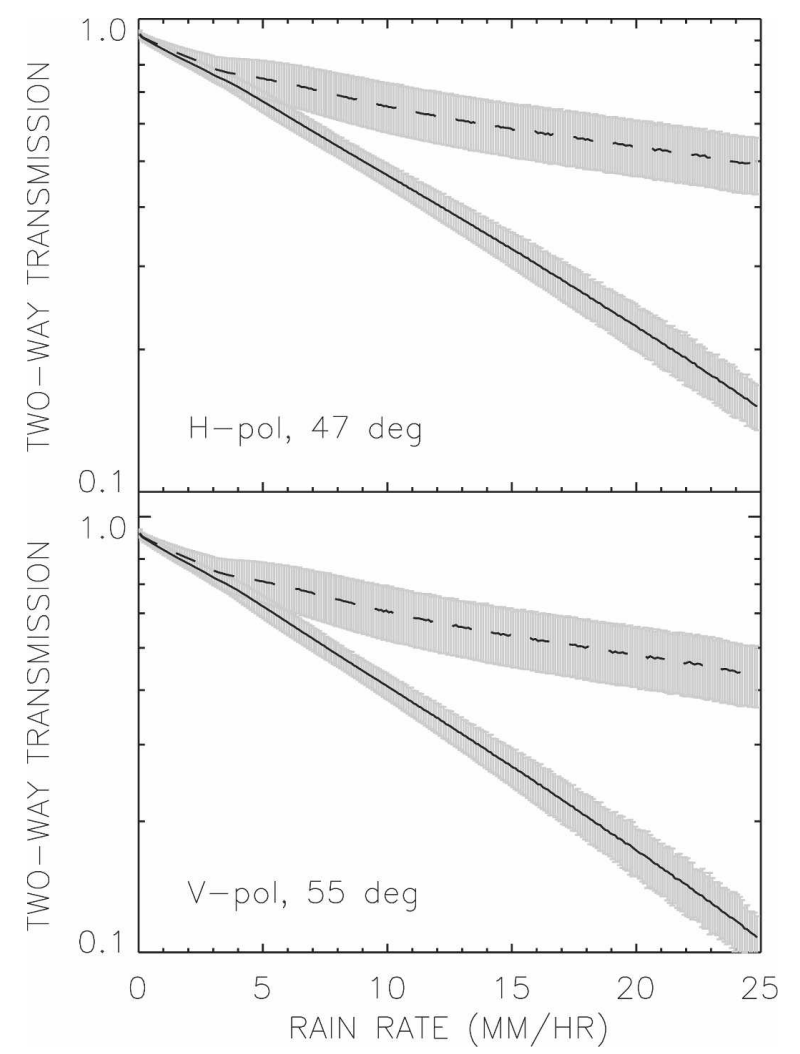

FIG. 2. The 13.4-GHz two-way transmission binned by retrieved rain rate when uniform beam filling is assumed (solid) and when nonlinear beam filling is allowed by directly calculating transmission from AMSR brightness temperatures (dashed). The gray envelope corresponds to one standard deviation.

attenuation measurement that does not vary as strongly with drop size distribution. Ultimately, the correction of an active scatterometer signal with passive radiometer information requires making connections between the parameters of the radar equation (for the active signal) and the radiative transfer equation (for the passive signal). Simulations using a discrete-ordinates solution to the radiative transfer equation (Stamnes et al. 1998) have shown us that the radar equation is a highly simplified and has a limited (but very accurate) approximation to the radiative transfer equation. In section 4a we will show how the parameters of these equations are related.

It is well known, thanks to the TRMM precipitation radar, that when a radar at an attenuating frequency is used to remotely sense through the vertical depth of a storm, the vertical profile of the hydrometeors can have a significant impact on the overall measured backscatter. Simplified simulations (Hilburn and Wentz 2003) have shown that the same amount of liquid water can produce backscatter values that differ by several decibels, depending upon the vertical distribution of the 
liquid water. The impact of the vertical profile variability on measured backscatter is discussed in section $4 \mathrm{~b}$.

Our technique for calculating volumetric backscatter is unique in that it relies on information from the effective temperature depression, which is discussed in section $4 \mathrm{c}$. The results of how our technique compares with established relationships from radar meteorology are discussed in section $4 \mathrm{~d}$.

\section{a. Drop size distribution variability}

Volumetric radar backscatter is typically estimated as a function of rain rate. It is well known that there is a large amount of scatter between these parameters because of raindrop size distribution variability. This is typically addressed by discriminating between "convective" and "stratiform" precipitation types and using relationships appropriate to each type of precipitation. Rather than using this technique, which accounts for a spectrum of variability by using a binary classification, we will attempt a different technique. In this subsection, we will derive a relationship between the volumetric radar backscatter and the parameters of the radiative transfer equation. This will allow us to then use additional radiometric information (section $4 \mathrm{c}$ ) to estimate the volumetric backscatter while accounting for drop size distribution variability.

We begin by noting that the radar reflectivity $\eta$ is related to the scattering coefficient $\left(k_{\text {sca }}\right)$ by

$$
\eta=\tilde{P} k_{\text {sca }}
$$

where $\tilde{P}$ indicates the relative strength of backscattering and is given by

$$
\tilde{P}=\frac{\int \sigma_{\mathrm{sca}} P(\pi) N d D}{\int \sigma_{\mathrm{sca}} N d D}=\frac{\eta}{k_{\mathrm{sca}}}
$$

where $\sigma_{\text {sca }}$ is the scattering cross section of a drop, $P(\pi)$ is the phase function in the backward direction, and $N$ is the number of raindrops per volume with diameters between $D$ and $D+d D$. Next, note that the extinction coefficient $\left(k_{\text {ext }}\right)$ is related to the scattering coefficient by

$$
\frac{k_{\mathrm{sca}}}{k_{\mathrm{ext}}}=a,
$$

where $a$ is the single scatter albedo of the drop ensemble. Therefore,

$$
\eta=\tilde{P} a k_{\mathrm{ext}} .
$$

Equation (12) is an exact relationship between the parameters of the radar equation $(\eta)$ and the radiative transfer equation $\left(k_{\text {ext }}, a\right.$, and $\left.\tilde{P}\right)$. Equation (12) already contains more unknowns than we can completely address with passive microwave data. Thus, we will combine two of the parameters. In the case of Rayleigh scattering $\tilde{P}$ takes a constant value of 1.5 . In the case of 13.4-GHz Mie scattering by an ensemble of spherical raindrops, $\tilde{P}$ varies from 1.2 to 1.8 , and is very strongly related to $a$. Thus, we define

$$
\omega=\left(\frac{\tilde{P}}{2}\right) a .
$$

Using Mie theory with a three-parameter gamma raindrop size distribution to simulate variability, we find that $\omega=0.90 a^{1.08}$ explains $99 \%$ of the variance at 13.4 GHz. This approximation is acceptable given the other uncertainties in the problem. Thus, the fundamental equation for volumetric backscatter is

$$
\eta=2 \omega k_{\mathrm{ext}} .
$$

Equation (14) is important because it reveals the functional form for volumetric backscatter. The volumetric backscatter is the product of $k_{\text {ext }}$, which is closely related to rain rate, with $\omega$, which is a measure of scattering. Equation (14) tells us that the variability between $z$ and $R$ is largely because of the variability between $\omega$ and $R$. Thus, our strategy will be to use the radiometer-derived attenuation to estimate $k_{\text {ext }}$, and the effective temperature depression to estimate $\omega$ (section $4 c)$.

\section{b. Vertical profile variability}

The volumetric rain backscatter is given by

$$
\sigma_{0, \mathrm{vbs}}=\int_{0}^{H \sec \theta}\left\{e\left[-2 \int_{0}^{l} k_{\mathrm{ext}}(l) d l\right]\right\} \eta(l) d l,
$$

where $k_{\text {ext }}$ and $\eta$ are functions of pathlength $l, H$ is column height, and $\theta$ is incidence angle. Equation (15) does not explicitly address horizontal inhomogeneity, although if $k_{\text {ext }}$ represents $\hat{k}_{\text {ext }}$ and $\eta$ represents $\hat{\eta}$, then (15) can be used for nonuniform situations (using the notation of section 3). Inhomogeneity is a problem when estimating a parameter only if the averaging operates on a strongly nonlinear function. For attenuation, the nonlinearity is the exponential function and so beam filling must be addressed to yield accurate results. We have addressed this in section 3. For the backscatter, the nonlinearity is much weaker and appears to be 
a less substantial problem (Hilburn and Wentz 2003), especially when compared with the other uncertainties in the problem.

Equation (15) can only be solved if the vertical profile of attenuation and backscatter are known. To understand and model the effect of the vertical profile variability on the volumetric backscatter let us first consider the simplest possible profile. If we assume that both $k_{\text {ext }}$ and $\eta$ are constant with height, we obtain

$$
\sigma_{0, \mathrm{vbs}}=\left(\frac{1-\tau^{2}}{2 k_{\mathrm{ext}}}\right) \eta=\left(1-\tau^{2}\right) \omega .
$$

The next step in the complexity of assuming that $k_{\text {ext }}$ and $\eta$ are linear functions of pathlength yields a problem of sufficient mathematically complexity as to offset any gains in physical understanding. Consider instead a layer approximation to (15). It is not difficult to show that the $N$-layer approximation to (15) is

$$
\sigma_{0, \mathrm{v} b s}=\sum_{i=1}^{N}\left(\prod_{j=0}^{i-1} \tau_{j}^{2}\right)\left(1-\tau_{i}^{2}\right) \omega_{i},
$$

where layer one is the uppermost layer ( so $\tau_{0}^{2}=1$ ) and layer $N$ is nearest to the ocean surface. Equation (17) is equal to (16) for $N=1$. Now consider the next step in complexity, $N=2$, for which (17) becomes

$$
\sigma_{0, \mathrm{vbs}}=\left(1-\tau_{1}^{2}\right) \omega_{1}+\tau_{1}^{2}\left(1-\tau_{2}^{2}\right) \omega_{2} .
$$

Now define "mean" and "perturbation" components of the profile:

$$
\begin{aligned}
& \bar{\omega}=\frac{\omega_{1}+\omega_{2}}{2}, \quad \omega^{\prime}=\frac{\omega_{1}-\omega_{2}}{2}, \\
& \bar{\tau}^{2}=\tau_{1}^{2} \tau_{2}^{2}, \quad \text { and } \quad \tau^{\prime 2}=\frac{\tau_{1}^{2}}{\tau_{2}^{2}},
\end{aligned}
$$

and (18) becomes

$$
\sigma_{0, \mathrm{vbs}}=\left(1-\bar{\tau}^{2}\right) \bar{\omega}+\left(1+\bar{\tau}^{2}\right) \omega^{\prime}-2 \bar{\tau}\left(\omega^{\prime} \tau^{\prime}\right) .
$$

The first term is the backscatter from the mean profile and is analogous to the constant profile (16). The second term provides more backscatter if the profile is "top heavy" $\left(\omega^{\prime}>0\right)$, such as in the "bright band" case of stratiform precipitation, and less if it is "bottom heavy" $\left(\omega^{\prime}<0\right)$, such as in a typical case of convective precipitation. Because the backscatter is attenuated at $\mathrm{Ku}$-band frequencies, the location of the maximum backscatter is important. Note that in the limit of a nonattenuating frequency (20) becomes $H \sec \theta \bar{\eta}$, and the profile shape does not modify the mean backscatter response. The third term represents the covariance between the absorption and scattering and is a function of the drop size distribution and phase of the particles.
The simplest possible model for the volumetric backscatter that includes vertical profile variability is Eq. (20). As we will discuss in the next section, the radiometer provides two pieces of information: an attenuation signal and a scattering signal. The attenuation signal is a function of the total amount of liquid present $\left(\bar{\tau}^{2}\right)$. The scattering signal is a function of the total amount of liquid $\left(\bar{\tau}^{2}\right)$, how this liquid is distributed among different size raindrops $(\bar{\omega})$, and how the liquid is distributed vertically in the column $\left(\tau^{\prime 2}\right.$ and $\left.\omega^{\prime}\right)$. We have three equations but five unknowns. Correcting scatterometer (active) data using a correction that relies upon radiometer (passive) data alone is an underdetermined problem, and unfortunately we cannot explicitly calculate everything. As discussed further in section $4 \mathrm{~d}$, while the functional form of our correction ignores vertical profile variability, it is implicitly included through the fitting procedure and the use of the effective temperature depression.

\section{c. Effective temperature depression}

As discussed in section 3, dual-polarization passive measurements can be used to decouple radiometric attenuation and scattering signals from precipitation. The attenuation signal is expressed in terms of the transmission $\tau^{2}$. The scattering signal has been typically expressed in terms of the polarization-corrected temperature (Spencer 1986; Spencer et al. 1989), and we choose to express it with an analogous quantity-the effective temperature depression $\Delta T_{e}$. The effective temperature depression is very similar to the scattering index of Petty (1994). The effective temperature depression represents the deviation induced by precipitation from the assumed relationship between the effective air temperature and the columnar water vapor and sea surface temperature in the absence of precipitation (Wentz and Spencer 1998). The effective temperature depression is usually negative because radiative scattering reduces the upwelling brightness temperature, and the effect is at most about $-30 \mathrm{~K}$ at $36.5 \mathrm{GHz}$. We use the $36.5-\mathrm{GHz}$ AMSR signal because the scattering signal at $18.7 \mathrm{GHz}$ is much smaller and the $36.5-\mathrm{GHz}$ signal is significantly less sensitive to ice than that at $89.0 \mathrm{GHz}$ (e.g., Petty 2001).

To estimate the volumetric backscatter and account for drop size distribution variability [(14)], we need estimates of $k_{\text {ext }}$ and $\omega$. The connection between $k_{\text {ext }}$ and the attenuation signal is already clear (e.g., Wentz and Spencer 1998). It seems reasonable that there might be some connection between $\omega$ and the scattering signal. To determine this, we performed simulations using a discrete-ordinates solution to the radiative transfer equation (Stamnes et al. 1998). We found that for a 
TABLE 1. Coefficients for the model [(21)].

\begin{tabular}{lcccc}
\hline \hline Polarization & $R$ & $R^{2}$ & $\Delta T_{e}^{2}$ & $\Delta T_{e}^{4}$ \\
\hline Vertical & $1.648 \times 10^{-3}$ & $-3.826 \times 10^{-5}$ & $5.125 \times 10^{-5}$ & $-2.550 \times 10^{-5}$ \\
Horizontal & $2.245 \times 10^{-3}$ & $-4.409 \times 10^{-5}$ & $9.715 \times 10^{-5}$ & $-4.017 \times 10^{-5}$ \\
\hline
\end{tabular}

vertically homogeneous layer of liquid, a close relationship exists between the 13.4-GHz single scatter albedo and the $36.5-\mathrm{GHz}$ effective temperature depression. A fourth-order polynomial in $\Delta T_{e}$ explains $93 \%$ of the variance. This suggests that, at least under idealized circumstances, a relationship does exist; and it suggests that it would not be unreasonable to use a polynomial to parameterize $\omega$ in terms of $\Delta T_{e}$ using the data.

We fit a polynomial in $\Delta T_{e}$ for $\omega$ using SeaWinds, AMSR, and NCEP data as described in section 4d. The coefficients for this polynomial are given in Table 1 . It is important to emphasize that the $\omega-\Delta T_{e}$ relationship given in Table 1 is empirical. Thus, the effects of ice are only implicitly included, and not explicitly addressed. For future work we plan to investigate multichannel approaches using $18.7,36.5$, and $89.0 \mathrm{GHz}$ to estimate waccounting for ice. It is important to realize that while $\omega-\Delta T_{e}$ is approximate, this is the first study to attempt to use other radiometric information to account for variability. Previous investigations (Stiles and Yueh 2002; Draper and Long 2004) have only used rain-rate information. We will now show that rain-rate information alone is insufficient, and we will also show that there is a systematic $\Delta T_{e}$ signal in the scatterometer measurements.

Figure 3 shows why rain rate alone is not sufficient. There is a nonmeteorological "doughnut" of high scatterometer winds shown in the upper-left panel (Fig. 3a). NCEP winds are shown for reference (Fig. 3b). Visually, one can see that AMSR rain rates (Fig. 3c) only match with the northern part of the feature and are not high enough to match the southern half of the feature. It is clear that the effective temperature (Fig. 3d) fills in the other important information. The reasons why the same amount of liquid might produce significantly different effects on the scatterometer winds could have to
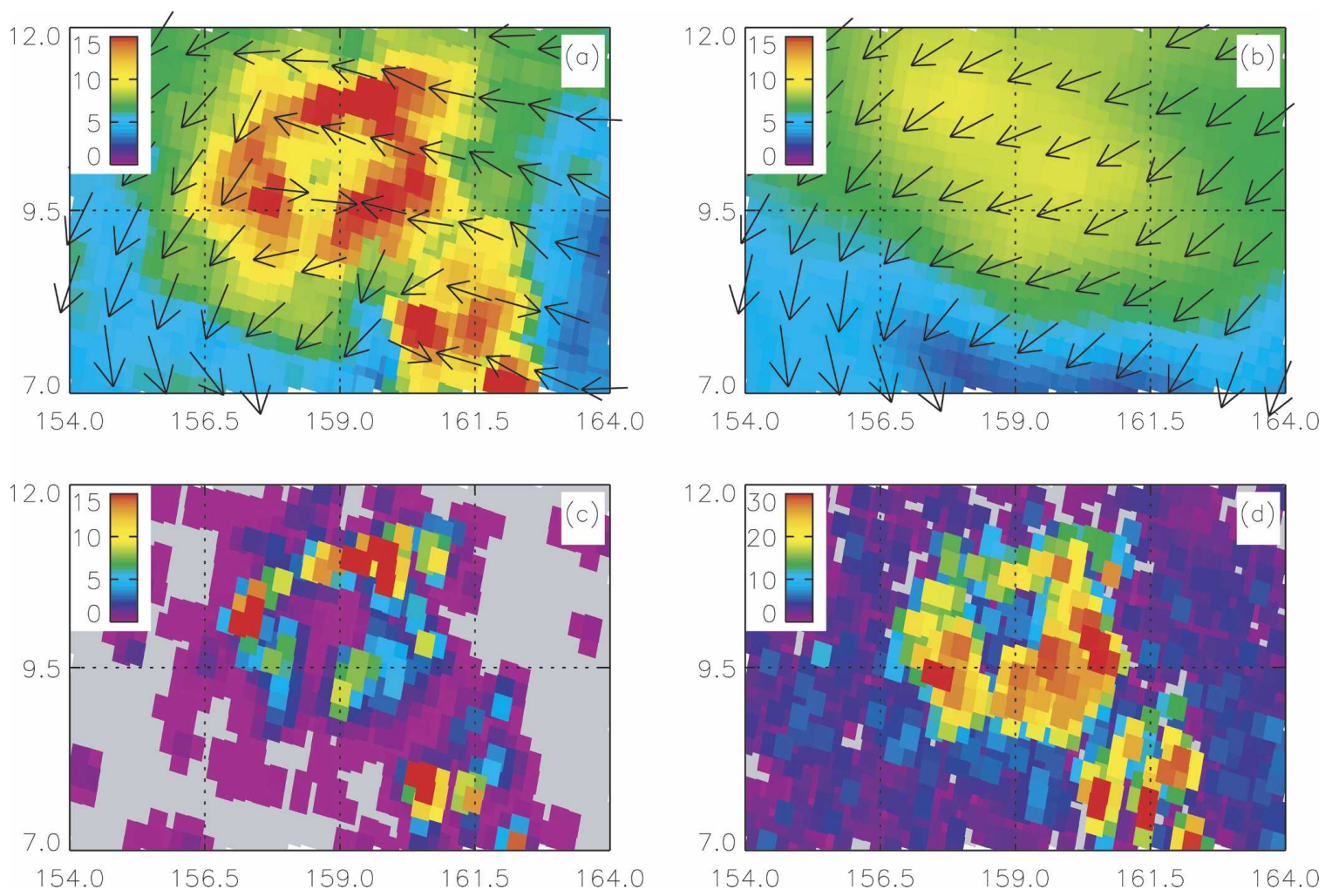

FIG. 3. (a) Uncorrected SeaWinds winds $\left(\mathrm{m} \mathrm{s}^{-1}\right)$, (b) NCEP winds $\left(\mathrm{m} \mathrm{s}^{-1}\right)$, (c) AMSR rain rate (mm $\mathrm{h}^{-1}$ ), and (d) AMSR effective temperature depression (multiplied by -1) (K) for a tropical rain example west of the Marshall Islands on 28 Jun 2003. 


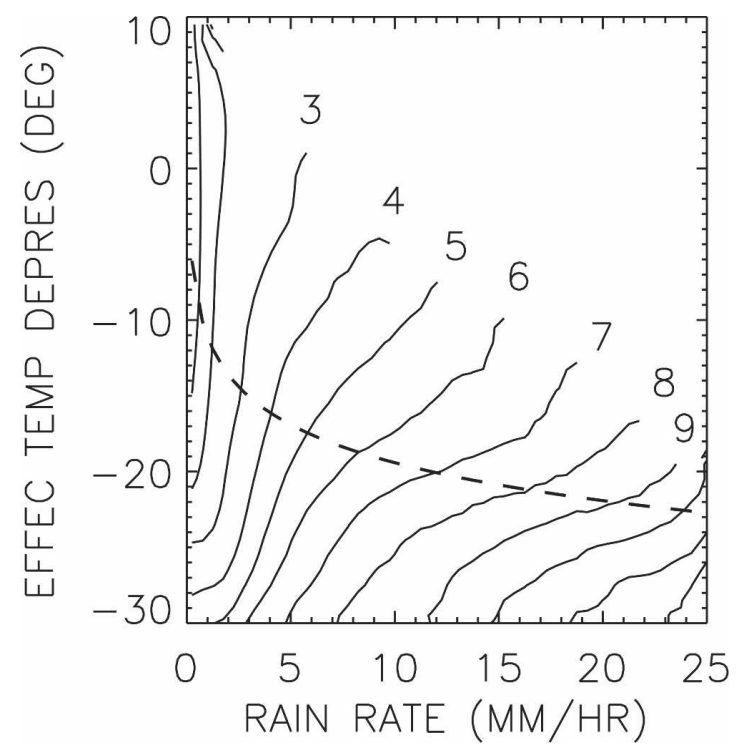

FIG. 4. SeaWinds minus NCEP wind speed vs rain rate and effective temperature depression. The contour interval is $1 \mathrm{~m} \mathrm{~s}^{-1}$, and the dashed line shows the average effective temperature depression vs rain rate.

do with drop size distribution or vertical distribution of liquid, which both depend on the type and age of the rain storm. As we have discussed (section 4b), it is not simply the amount of liquid, but how it is distributed among differently sized drops and how it is distributed vertically that determines the amount of backscattered power. Figure 3 is a good qualitative example showing that the effective air temperature is correlated with these factors.

Further evidence showing the importance of the effective temperature depression in a potential rain correction is given in Fig. 4. We see that the difference between SeaWinds and NCEP winds increases with increasing rain rate and with decreasing effective air temperature. The wind difference contours are nearly vertical at low rain rates, consistent with the fact that drop size distribution and vertical profile effects are small at low rain rates. Above $2 \mathrm{~mm} \mathrm{~h}^{-1}$ the wind difference contours become increasingly horizontal. It is obvious that a correction based on rain rate alone will miss much of the variability. Figure 4 also shows the average relationship between $\Delta T_{e}$ and $R$, which is fit well by $-\Delta T_{e}=3.61 \times \ln (R)+11.1$. Please note this equation is given for reference and is not used by the algorithm.

There were certain cases in which the $\omega-\Delta T_{e}$ relationship seemed to produce winds that were overcorrected. In high-latitude storms, there were some cases in which the rain rate was low, and the difference between SeaWinds and NCEP was low, but $\Delta T_{e}$ was high. In these cases the rain correction left patches of low

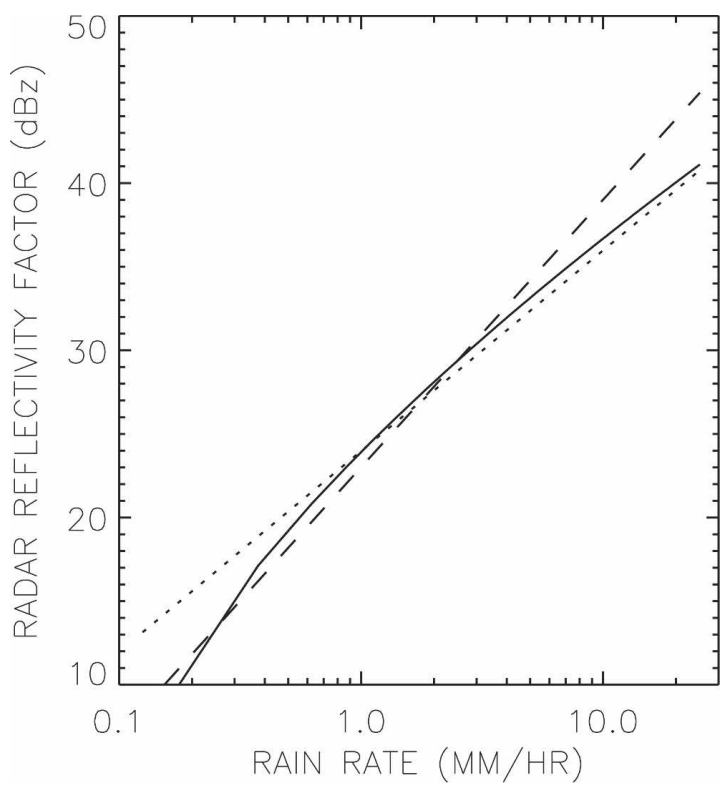

FIG. 5. The volumetric backscatter converted into radar reflectivity factor $(z)$ for horizontal polarization. Shown are our derived relationship (solid), the Marshall-Palmer relationship (dashed), and the National Weather Service tropical relationship (dotted). While our relationship is plotted here vs rain rate, the actual functional form within the model depends on the effective temperature depression.

winds that did not resemble meteorological features. Presumably, these were cases in which the ratio of ice water to rainwater was high, so that the effective temperature depression was due mostly to ice, and the scatterometer was not strongly affected. In these cases, the retrieved winds were generally good without any correction being necessary. Thus, to avoid overcorrecting in these situations, we do not apply our correction in cases in which the model function fits the data well (section 6). This was very effective at screening these cases, and it means that no correction is performed on slightly less than $1 \%$ of the data with AMSR rain rates greater than zero.

\section{d. Volumetric backscatter model}

Combining (16) with (1), and assuming that rain roughening depends on rain rate, we obtain our full rain correction model:

$$
\sigma_{0, \text { meas }}-\tau^{2} \sigma_{0, \text { wind }}=\tau^{2} f_{1}(R)+\left(1-\tau^{2}\right) f_{2}\left(\Delta T_{e}\right) .
$$

The transmission is a weighting function between contributions from rain roughening and volumetric backscatter. Thus, we have separated the roughening and backscatter terms and we can use a least squares tech- 


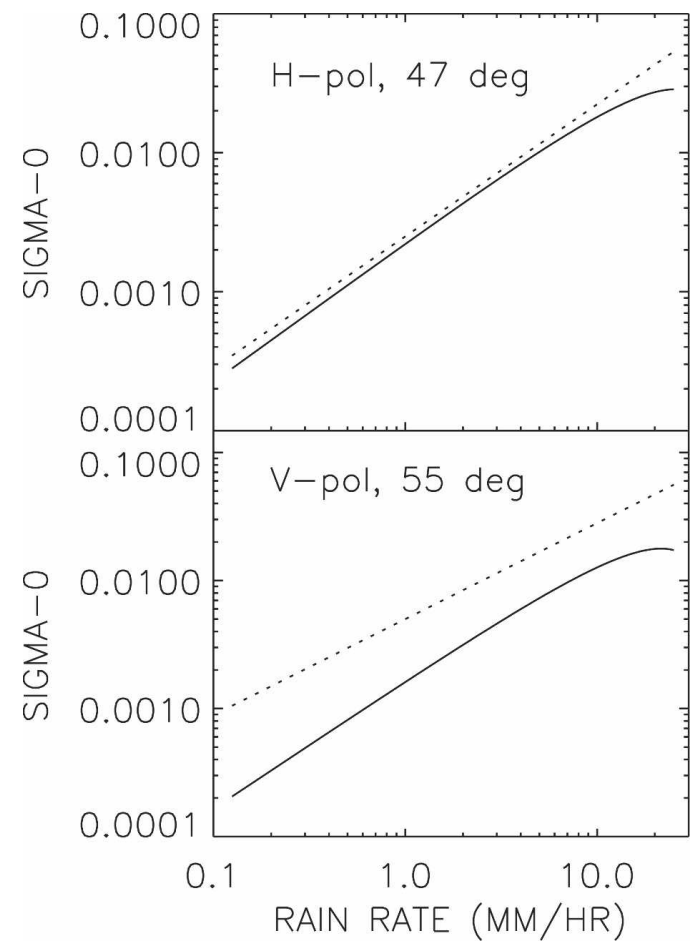

FIG. 6. Magnitude of the rain-roughening term as found by our model (solid) vs the magnitude found by Contreras et al. (2003) (dotted). The Contreras et al. values have been adjusted to SeaWinds incidence angles and a $5 \mathrm{~m} \mathrm{~s}^{-1}$ wind speed (typical speed in their study) has been removed.

nique to simultaneously solve for both terms. In fitting (21) to SeaWinds and AMSR data, we assume a second-order polynomial in $R$ for $f_{1}$ and a fourth-order polynomial in $\Delta T_{e}$ for $f_{2}$. NCEP wind speeds and directions were used with SeaWinds geometry to specify a value of $\sigma_{0 \text {,wind }}$. The values of the coefficients are given in Table 1. The rain-roughening term will be discussed in the next section, but first we discuss the backscatter term.

Although our volumetric backscatter term is a function of $\Delta T_{e}$ and not $R$, and although $f_{2}$ is related to the pseudo-single scatter albedo and not the radar reflectivity factor, it is informative to convert our correction into a $z-R$ relationship in order to compare it with other expressions from radar meteorology. Using the relationship between the radar reflectivity $\eta$ and the radar reflectivity factor $z$ (Doviak and Zrnić 1993), (14) becomes

$$
z=\frac{2 \omega k_{\mathrm{ext}}}{\pi^{5}\left|K_{w}\right|^{2} / \lambda^{4}}
$$

where $\lambda$ is the wavelength of SeaWinds and $\left|K_{w}\right|^{2}$ depends on the complex index of refraction. To compare

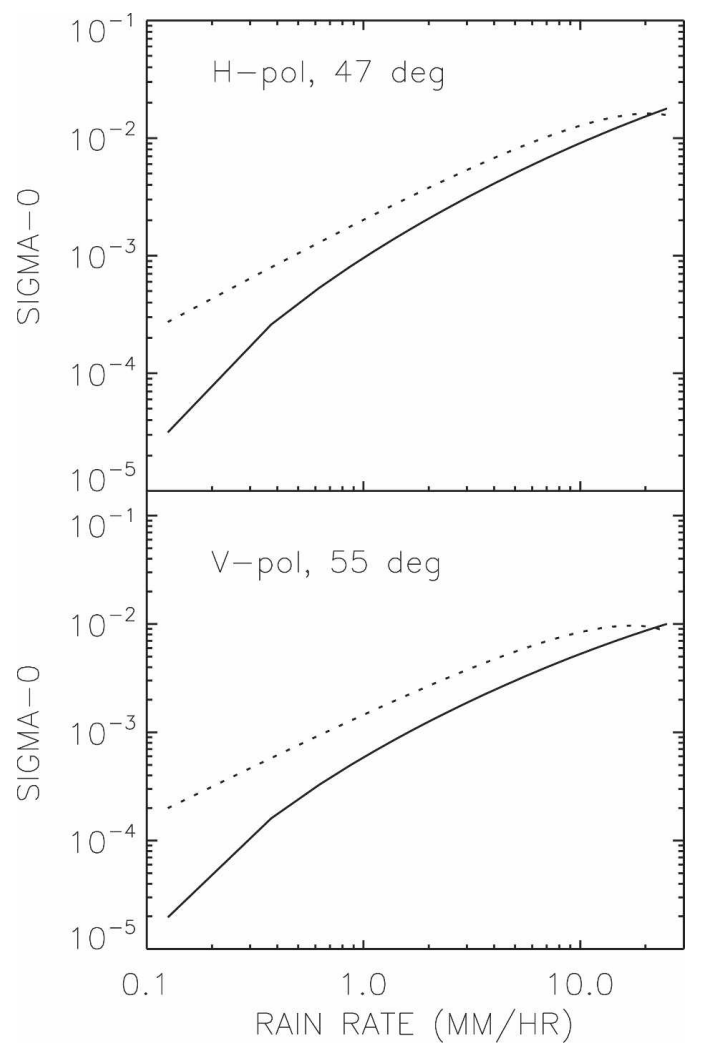

FIG. 7. Relative contributions of volumetric backscatter (solid) and rain roughening (dotted) to the rain effect in our model.

our results with standard $z-R$ relationships, in which the radar beam is more-or-less uniformly filled, (22) should be evaluated using the value of $k_{\text {ext }}$ that is appropriate for a uniformly filled beam. Figure 5 shows that the $z-R$ relationship implied by our correction matches well with the Marshall-Palmer relationship ( $z$ $=200 R^{1.6}$ ) at rain rates below $3 \mathrm{~mm} \mathrm{~h}^{-1}$, but more closely follows the National Weather Service tropical relationship $\left(z=250 R^{1.2}\right)$ (Belville 1999) at higher rain rates. Theoretical work using linear vertical profiles indicates that vertical profile effects become noticeable as rain rates exceed $3 \mathrm{~mm} \mathrm{~h}^{-1}$ (Hilburn and Wentz 2003). We believe this change in slope around $3 \mathrm{~mm} \mathrm{~h}^{-1}$ is indicative of implicitly included vertical profile effects. The decrease in slope as rain rate increases is consistent with bottom-heavy profiles predominating as rain rate increases. This is consistent with convective profiles being associated with higher average rain rates (Liu and Fu 2001).

Fitting power laws to our curve in Fig. 5 gives $z_{H}=$ $226 R^{1.29}$. Figure 5 plots the horizontal polarization relationships because $z-R$ relationships are historically suited for horizontal polarization radar systems. The fit for vertical polarization gives $z_{V}=118 R^{1.28}$. From 


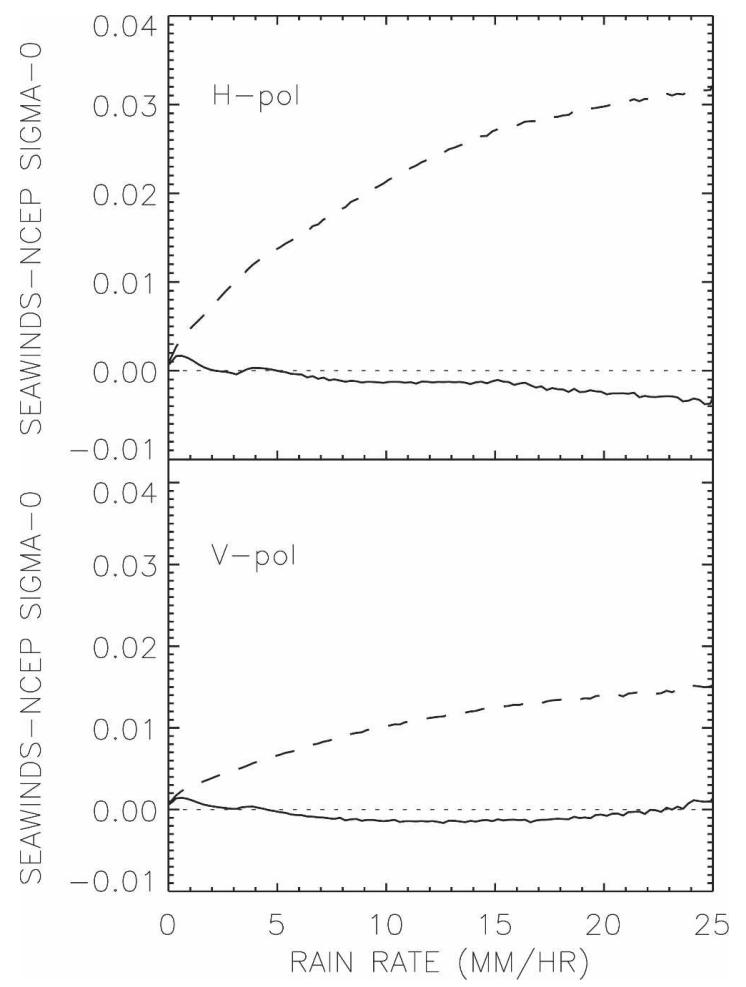

FIG. 8. SeaWinds minus the attenuated NCEP radar cross section vs rain rate showing the uncorrected (dashed) and corrected (solid) values. The zero difference line is dotted. Figures 8-13 make use of the entire SeaWinds in the Midori-II dataset.

this we see that our radar reflectivity factor for vertical polarization is consistently about $3 \mathrm{~dB}$ higher than for horizontal polarization. This is somewhat high because we would expect this difference to vary from 0.5 to $1.5 \mathrm{~dB}$ over the rain-rate range in Fig. 5 (Timothy et al. 1999).

\section{Estimating surface roughening}

The effects of raindrop collisions with the ocean surface on radar cross sections is complicated and poorly understood. It is fairly certain however that at these large incidence angles and at $\mathrm{Ku}$-band frequencies, rain roughening increases both the vertically and horizontally polarized surface backscatter (Contreras et al. 2003). Early on, we found that the rain effect at low rain rates is too large to be because of volumetric backscattering alone, and a backscatter-only model would imply $z-R$ relationships far outside of what is considered typical in radar meteorology. The model developed in section [Eq. (21)] allows for simultaneous solution of both the rain roughening and the volumetric backscatter.

The rain roughening should depend on some moment of the raindrop size distribution at the sea surface.

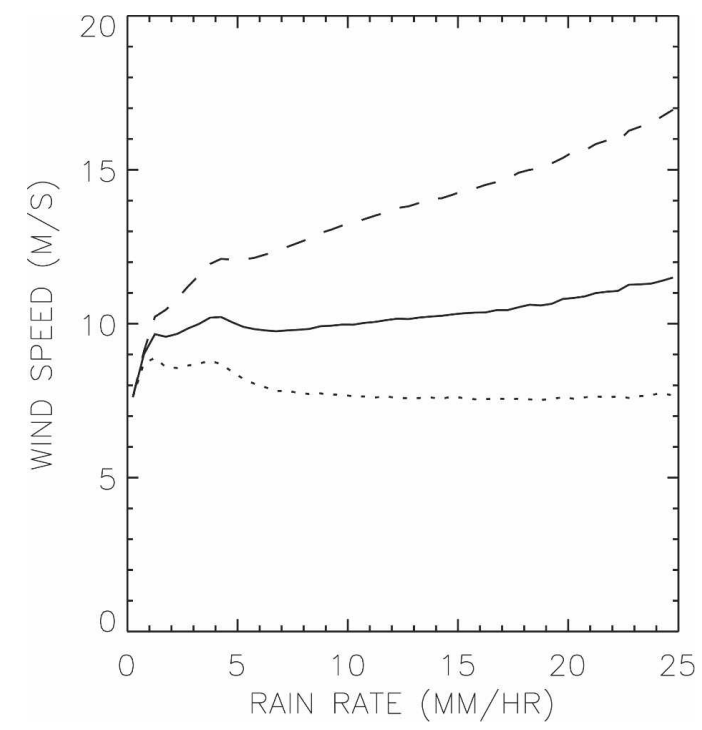

FIG. 9. Uncorrected (dashed), corrected (solid), and NCEP (dotted) wind speeds vs rain rate.

The most natural moment to choose is rain rate. We assumed rain roughening could be parameterized as a second-order polynomial in rain rate in order to account for deviations from linearity. Our relationships are shown in comparison with those of Contreras et al. (2003) in Fig. 6. We have adjusted the Contreras et al. relationships to SeaWinds incidence angles and a 5 $\mathrm{m} \mathrm{s}^{-1}$ wind speed has been removed (the typical wind speed in their study). Note that the Contreras et al. (2003) results are mainly for low winds. Our horizontal polarization curve matches well with Contreras et al., while the vertical polarization curve is about $2-3 \mathrm{~dB}$ lower. Power-law fits through our model-derived values give $\Delta \sigma_{0, \text { rain }}=0.0018 R^{0.79}$ for vertical polarization and $\Delta \sigma_{0, \text { rain }}=0.0024 R^{0.83}$ for horizontal polarization. It is interesting to note that relative to horizontal polarization, the vertical polarization rain-roughening terms and backscatter terms are both smaller than would be expected. It is unclear why the vertical polarization rain effect is consistently smaller than expected.

Figure 7 shows how the rain-roughening contribution in our model compares with the volumetric backscatter contribution; by "contribution" we mean multiplied by the appropriate function of transmission [as in Eq. (21)]. We see that in terms of actual contribution to the rain effect, the rain-roughening term makes a larger contribution. While the quasi-orthogonal nature of rain roughening and volumetric backscatter means that some of the backscatter signal could have been aliased into the roughening signal, the rain roughening is already smaller than the values found by Contreras et al. (2003). Draper and Long (2004) found that surface 


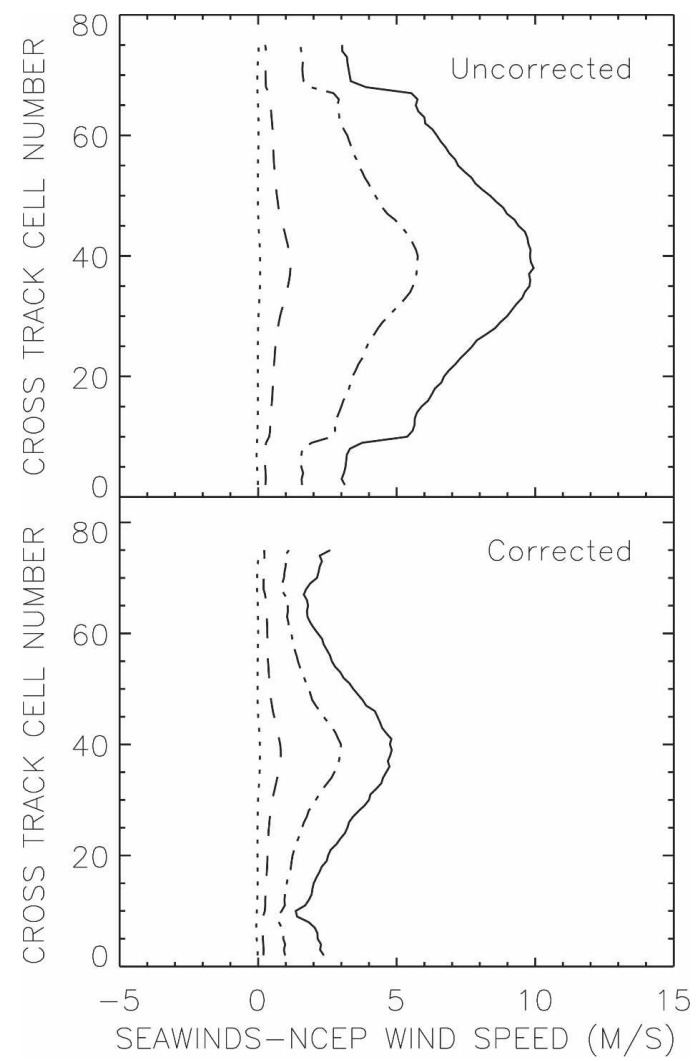

FIG. 10. (top) Uncorrected and (bottom) corrected SeaWinds minus NCEP wind speeds vs cross-track cell number for no-rain (dotted), light rain (dashed, $0-2.5 \mathrm{~mm} \mathrm{~h}^{-1}$ ), moderate rain (dashdot, 2.5-7.5 $\mathrm{mm} \mathrm{h}^{-1}$ ), and heavy rain (solid, 7.5-25 $\mathrm{mm} \mathrm{h}^{-1}$ ) cases.

roughening was more (less) important than volumetric backscatter at low (high) rain rates. They found the transition point to be between 10 and $100 \mathrm{~mm} \mathrm{~h}^{-1}$. Our results are consistent with their findings and we find the transition point to be in the $20-25 \mathrm{~mm} \mathrm{~h}^{-1}$ range.

\section{Corrected wind retrievals}

Figure 8 shows that our rain correction $[(21)]$ removes the systematic difference between the SeaWinds and the attenuated NCEP-derived radar cross sections. The nonlinear nature of the wind retrieval process means that the retrieved winds may still differ from NCEP winds. Figure 9 shows that all three (uncorrected SeaWinds, corrected SeaWinds, and NCEP) datasets all make small initial jumps as it begins raining, but the corrected SeaWinds and NCEP datasets level out while the uncorrected SeaWinds dataset continues to increase. The corrected winds are about $5 \mathrm{~m} \mathrm{~s}^{-1}$ lower than the uncorrected winds for moderate to heavy rain, but they are still about $3 \mathrm{~m} \mathrm{~s}^{-1}$ higher than those of NCEP. Because the scatterometer is capable of observ-

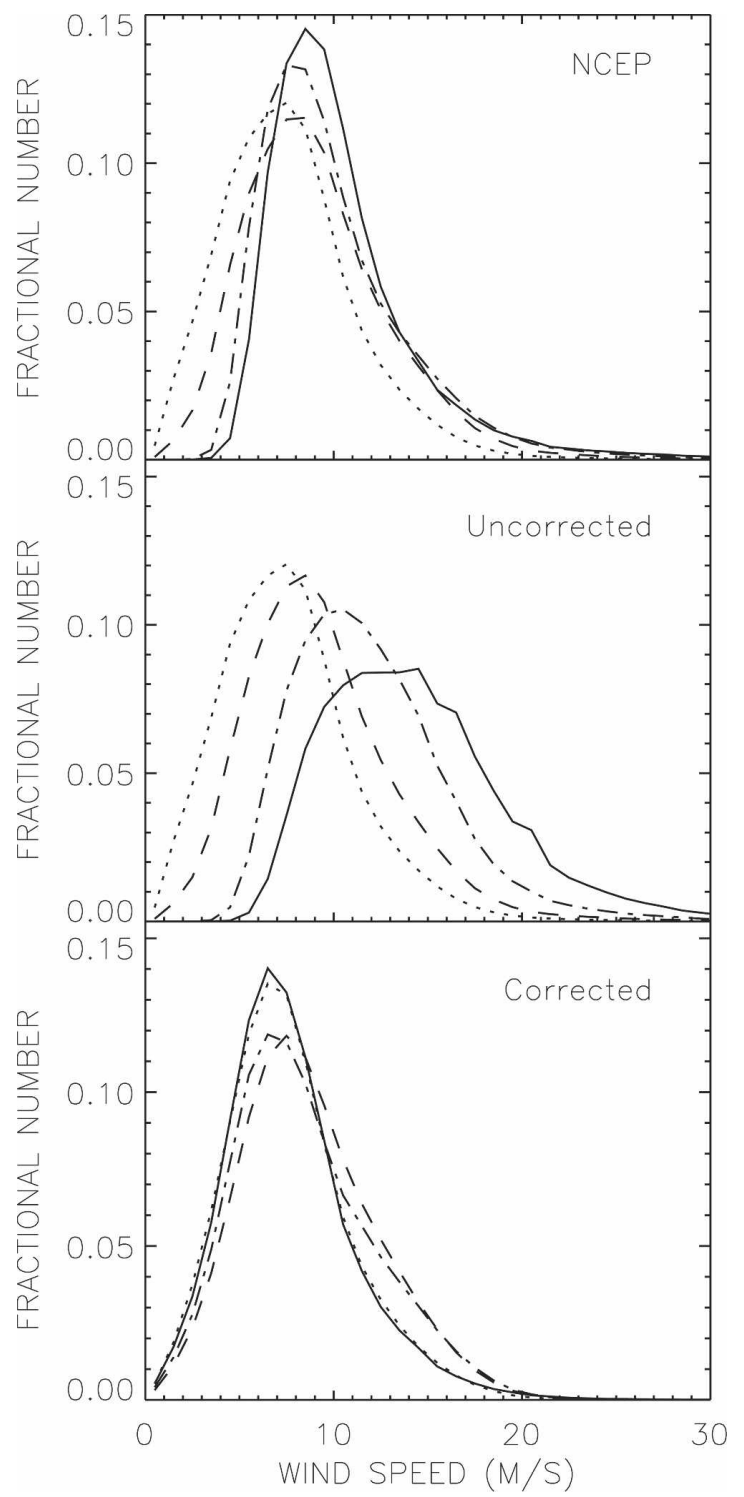

FIG. 11. (top) NCEP, (middle) uncorrected, and (bottom) corrected wind speed histograms for no-rain (dotted), light rain (dashed, 0-2.5 $\mathrm{mm} \mathrm{h}^{-1}$ ), moderate rain (dash-dot, 2.5-7.5 mm $\mathrm{h}^{-1}$ ), and heavy rain (solid, $7.5-25 \mathrm{~mm} \mathrm{~h}^{-1}$ ) cases.

ing mesoscale wind patterns that would not be in NCEP, the fact that our corrected winds are higher than those of NCEP for rain rates greater than $2 \mathrm{~mm} \mathrm{~h}^{-1}$ is not necessarily a sign that their quality is bad. The nonlinearity in the wind vector retrieval combined with the variable sampling across the scatterometer swath causes a nonconstant cross-swath wind speed bias in the presence of rain. This bias is shown in Fig. 10, and we see that the corrected winds greatly reduce the bias. This cross-swath bias is not present in the $\sigma_{0}$ data (not shown), but is an artifact of the nonlinearity and variable sampling in the wind vector retrieval. The applied 


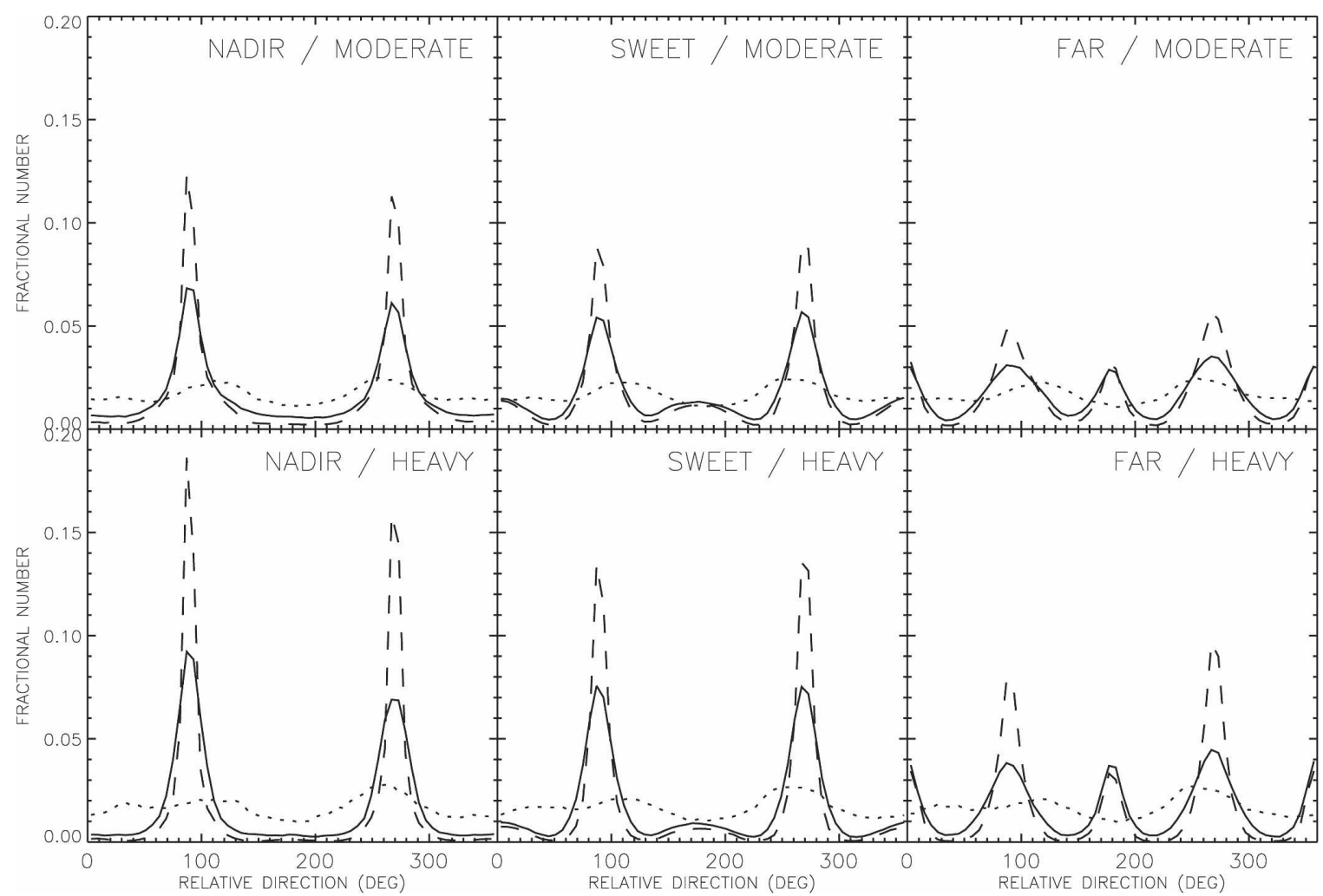

FIG. 12. NCEP (dotted), uncorrected (dashed), and corrected (solid) relative wind direction histograms for moderate and heavy rain in the nadir, sweet, and far portions of the swath. The direction is relative to the satellite track. The "nadir" is cells 31-46, the "far" swath is cells 1-9 and 68-76, and the remaining cells are the "sweet" portion of the swath. Light rain cases have a small effect and are not shown.

rain correction produces a great improvement in wind speed histograms (Fig. 11). NCEP wind speed histograms for different AMSR rain-rate bins all roughly overlap. The uncorrected wind speeds show a clear shift with rain rate in both the mode and the amount of weight in the tails. Wind direction histograms are much improved (Fig. 12) with the cross-track wind peaks reduced by $50 \%$. Improvements in wind direction retrieval are an encouraging sign that the correction is indeed correctly modeling the problem properly. The retrievals have been made using the Ku-2001 geophysical model function, which is somewhat prone to retrieving cross-track directions in rain. A model function similar to Ku-2001, but with less of a tendency for crosstrack retrievals, is in development at Remote Sensing Systems. Figure 13 shows the geographical distribution of the improvements. The correction reduces the fractional difference with NCEP by as much as $50 \%$ in tropical locations and greatly reduces middle- and highlatitude differences. The corrected winds are higher than those of NCEP in the midlatitude Southern Hemisphere, because they are in nonraining conditions as well.
While the correction makes a positive statistical impact, an uncomfortably large number of cross-track directions still remain. One might be tempted to blame the correction; however, there are several practical issues that limit how well any correction can perform. As discussed in section 2, there are four reasons as to why the scatterometer and radiometer are capturing different scenes: 1) SeaWinds makes both forward and backward looks while AMSR looks only forward, 2) the SeaWinds horizontal polarization (inner) beam has a different incidence angle than that of AMSR, 3) the SeaWinds footprint is about twice as large as the 36.5$\mathrm{GHz}$ AMSR footprint, and 4) because rain is variable on scales smaller than the instrument footprints and because the footprints are not centered on the same positions on the earth, there are beam mismatch issues arising from the complicated interaction between subpixel variability and the antenna beam patterns (Tournadre and Quilfen 2003). Adding even relatively small amounts of noise into the nonlinear wind retrieval process tends to cause the directions to assume cross-swath angles.

We make note of a few special cases encountered in 


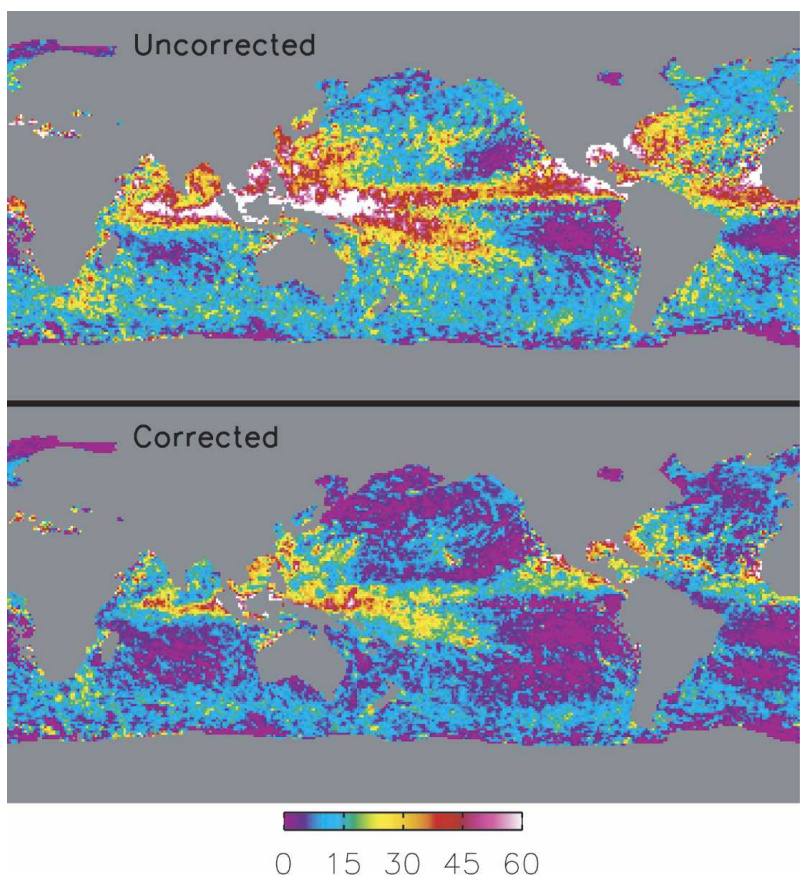

FIG. 13. Fractional difference (\%) between (top) uncorrected and (bottom) corrected SeaWinds wind speed vs NCEP. These maps only use wind vectors for which AMSR indicates a rain rate greater than zero.
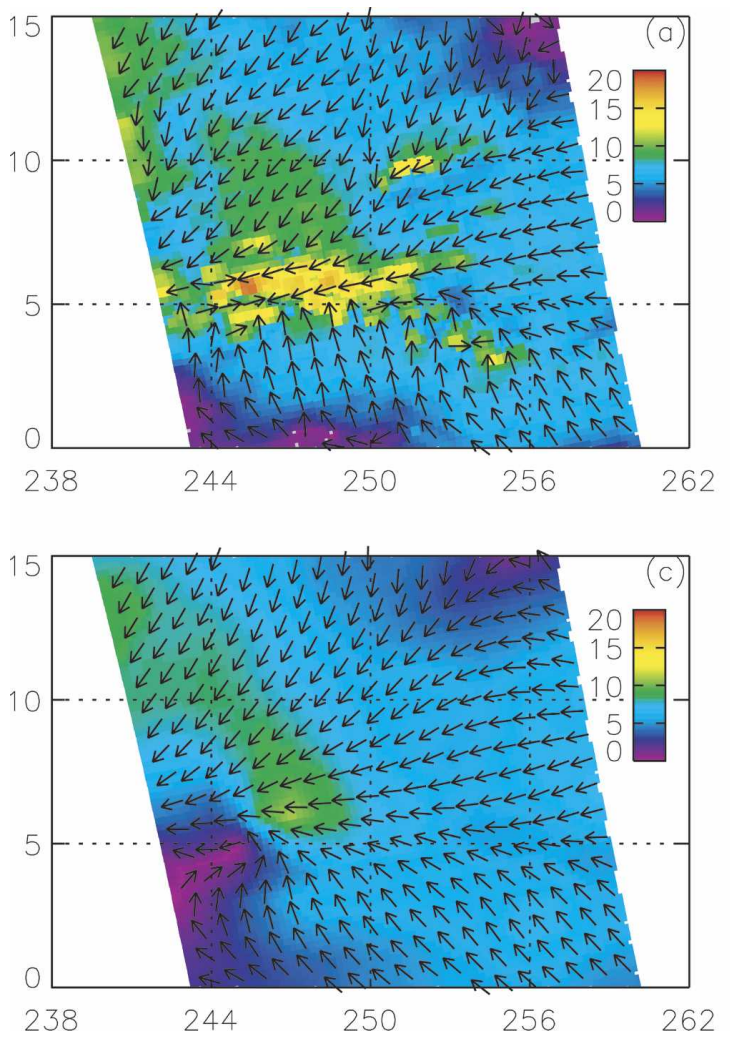

performing the wind retrieval on the corrected radar cross sections. First, many wind vector cells have more $\sigma_{0}$ values than are needed for the retrieval, which allows us to discard the most rain-contaminated $\sigma_{0}$ values ( $\sigma_{0}$ values for which $R>2.5 \mathrm{~mm} \mathrm{~h}^{-1}$ ). Doing this removes $7 \%$ of rain-contaminated $\sigma_{0}$ values prior to retrieval. Second, the correction causes $3 \%$ of the raincontaminated $\sigma_{0}$ values to become negative as a result of the correction. Typically, these are cases in which the measured $\sigma_{0}$ are small and the best results come from resetting the $\sigma_{0}$ back to their original values. Some wind vector cells are identified as being "rain contaminated" by AMSR observations, while the $\sigma_{0}$ values in the cell fit the model function very well with a small residual. We find that not correcting the $\sigma_{0}$ values works best in this situation and as a result $0.7 \%$ of the raincontaminated $\sigma_{0}$ values do not receive a correction.

Considering specific examples helps to illustrate the strengths and weaknesses of our rain correction procedure. Figure 14 demonstrates the performance of the correction on a tropical rain example. Wind speeds are much improved, but some wind directions still have problems. A midlatitude frontal example is given in Fig. 15. The wind speeds are reduced overall, and it is dif-
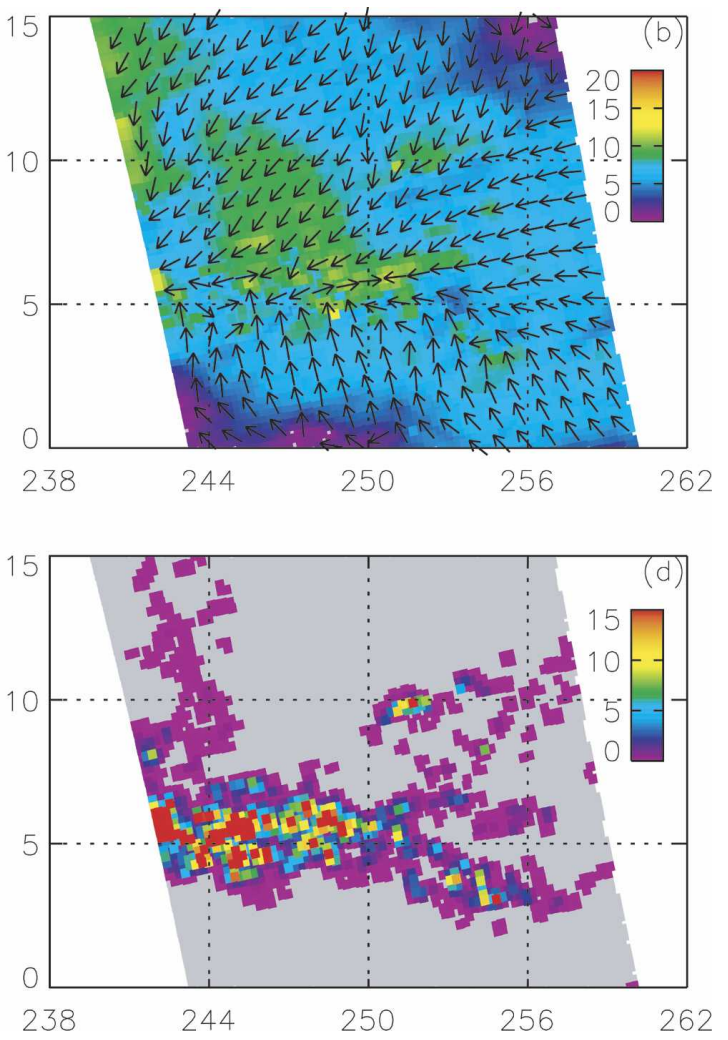

FIG. 14. Tropical convective rain off the western Central American coast on 10 Apr 2003. Shown are the (a) uncorrected SeaWinds, (b) corrected SeaWinds, (c) NCEP, and (d) AMSR rain rates. Units are meters per second for wind and millimeters per hour for rain. 

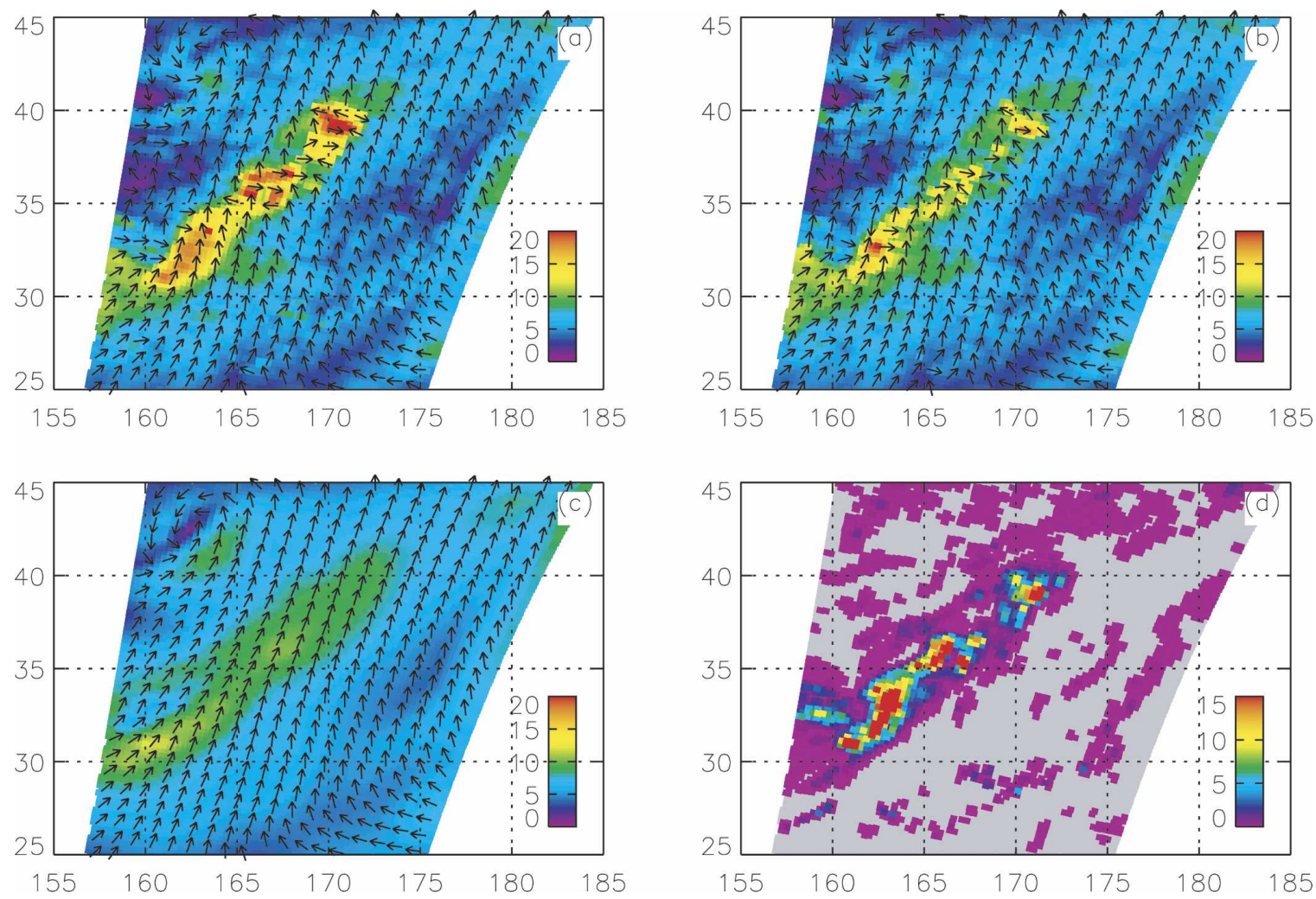

FIG. 15. As in Fig. 14, but for midlatitude frontal rain northwest of the Hawaiian Islands on 28 Jun 2003.

ficult to say if the remaining high winds are undercorrected or if the data represent true high winds along a front that are not depicted by NCEP. The geographical shape of the higher wind features look suspiciously like the shape of rain and suggest the winds are undercorrected. Hurricane Fabian is shown in Fig. 16. In this example the correction actually increased the wind speeds, from 44 (uncorrected) to 51 (corrected) $\mathrm{m} \mathrm{s}^{-1}$ at 1500 UTC. The National Hurricane Center (NHC) 1-min-sustained maximum wind based on a dropsonde at 1730 UTC was reported as being $57 \mathrm{~m} \mathrm{~s}^{-1}$. This 1-min mean is converted to the scatterometer 10-min mean using the U.S. Navy reduction factor of 0.88 , yielding a wind speed of $50 \mathrm{~m} \mathrm{~s}^{-1}$. Tropical storm examples are very sensitive to the attenuation correction, and the fact that our correction increases the wind speed to a reasonable value is evidence that our attenuation correction is working properly. It is interesting to note that the rain correction makes the hurricane structure more asymmetric and more similar to the Hurricane Research Division H-wind analysis field (see information online at http://www.aoml.noaa.gov/hrd/ data_sub/wind.html). The H-wind analysis in this case did not assimilate scatterometer data. It is clear from these examples that although the corrected wind dataset shows considerable improvements, caution should still be exercised in interpreting specific examples of these winds in the presence of heavy rain. The corrected winds should not be accepted blindly, but the radiometer rain information provided along with the corrected winds should be considered.

\section{Conclusions}

A model for the effects of rain on scatterometer data is proposed. The model accounts for attenuation, rain roughening of the sea surface, and volumetric backscatter. The attenuation is calculated directly from the radiometer data, while the rain roughening and volumetric backscatter are determined by fitting the model to data. Our rain roughening and volumetric backscatter both compare well with other published work, especially for horizontal polarization. The vertical polarization rain roughening and volumetric backscatter are both slightly lower than what other investigators have found. Beam-filling and drop size distribution variability are explicitly addressed. Explicit inclusion of vertical profile variability creates an underdetermined problem; however, the unique form of our correction has allowed it to be implicitly included. 

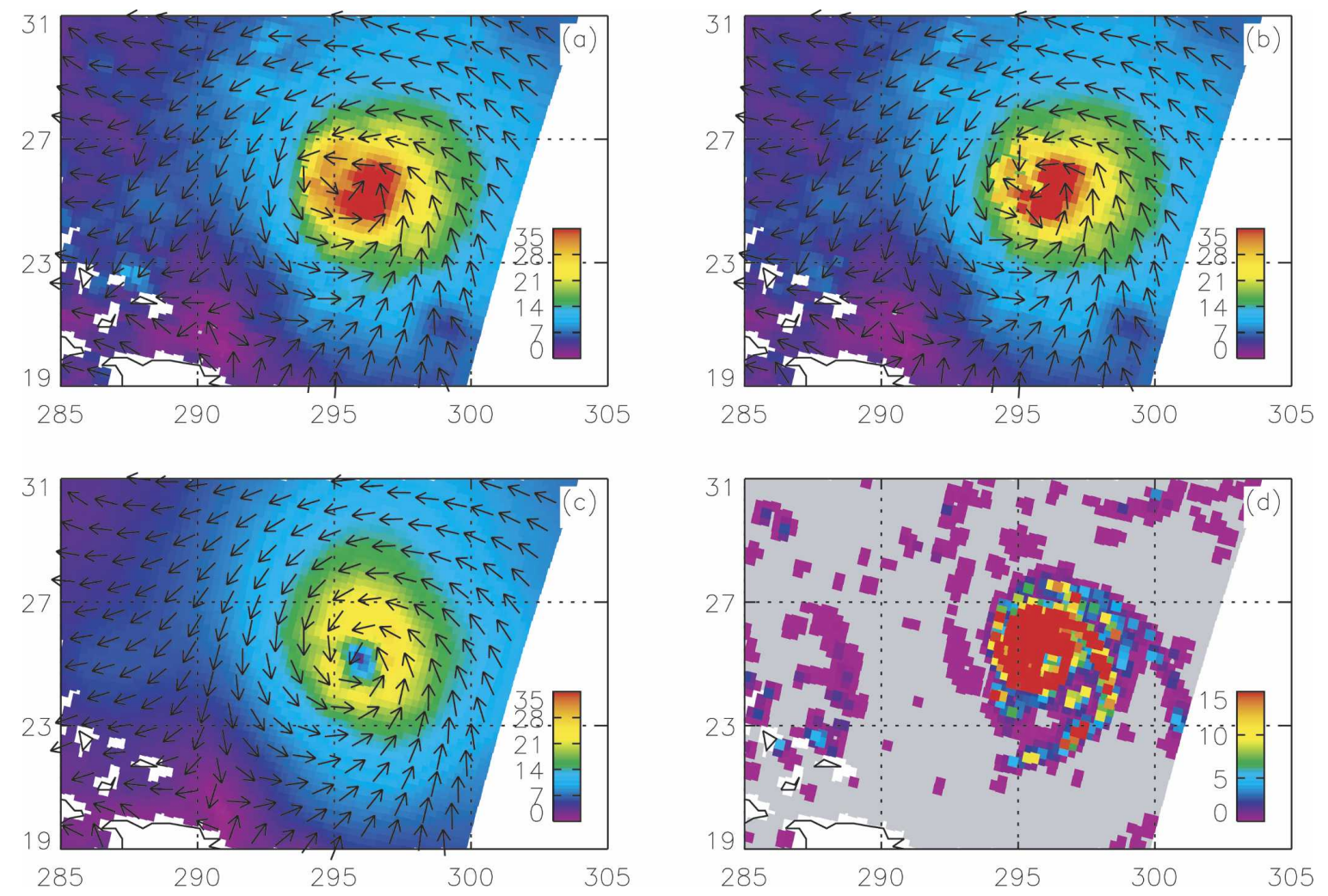

FIG. 16. As in Fig. 14, but for Hurricane Fabian on 1500 UTC 4 Sep 2003. The maximum uncorrected wind is $44 \mathrm{~m} \mathrm{~s}^{-1}$ and the maximum corrected wind is $51 \mathrm{~m} \mathrm{~s}^{-1}$.

The correction makes substantial improvements in wind speed with more modest improvements in wind direction. Both statistics and specific examples show these improvements. The correction is limited by the mismatch between the scatterometer and radiometer observations as discussed in sections 2 and 6 . The extreme spatial inhomogeneity of rain is the root of these issues. Our future efforts will be focused on obtaining the best possible footprint matching to minimize mismatch from rain inhomogeneity and obtain the best possible rain correction. The current work has clearly shown that the synergy of an active-passive sensor combination is able to provide more information than either instrument is able to provide by itself.

Acknowledgments. We are very grateful for continuing support from NASA. This research was supported by Jet Propulsion Laboratory Contract 1215789 under NASA Prime Contract NAS7-03001. Research results have been obtained through cooperation between Remote Sensing Systems and the Japanese Aerospace Exploration Agency (JAXA) in ADEOS-II research activity, and we are thankful to JAXA for making the Level-1A AMSR on Midori-II data available to us. We thank an anonymous reviewer whose comments enhanced the clarity of this paper.

\section{REFERENCES}

Belville, J. D., 1999: Recommended parameter changes to improve WSR-88D rainfall estimates during cool season stratiform rain events. NWS Memo., 3 pp.

Bourassa, M. A., D. M. Legler, J. J. O’Brien, and S. R. Smith, 2003: SeaWinds validation with research vessels. J. Geophys. Res., 108, 3019, doi:10.1029/2001JC001028.

Contreras, R. F., W. J. Plant, W. C. Keller, K. Hayes, and J. Nystuen, 2003: Effects of rain on Ku-band backscatter from the ocean. J. Geophys. Res., 108, 3165, doi:10.1029/ 2001JC001255.

Doviak, R. J., and D. S. Zrnić, 1993: Doppler Radar and Weather Observations. 2d ed. Academic Press, 562 pp.

Draper, D. W., and D. G. Long, 2004: Evaluating the effect of rain on SeaWinds scatterometer measurements. J. Geophys. Res., 109, C02005, doi:10.1029/2002JC001741.

Freilich, M. H., 1996: SeaWinds algorithm theoretical basis document. ATBD-SWS-01, Jet Propulsion Laboratory, California Institute of Technology, $56 \mathrm{pp}$. [Available online at http:// podaac.jpl.nasa.gov/quikscat/qscat_doc.html.]

Hilburn, K. A., and F. J. Wentz, 2003: Progress towards a combined AMSR/SeaWinds algorithm. SeaWinds Cal/Val Meeting, Arcadia, CA, Ocean Vector Wind Science Team, 1-24. 
[Available online at http://www.remss.com/presentations/ seawinds/hilburn_wentz_seawinds_sep2003_arcadia.pdf.]

Hoffman, R. N., C. Grassotti, and S. M. Leidner, 2004: SeaWinds validation: Effect of rain as observed by East Coast radars. $J$. Atmos. Oceanic Technol., 21, 1364-1377.

ITU Radiocommunication, 1992: Specific attenuation model for rain for use in prediction methods. ITU-R Recommendation P.838, 2 pp. [Available online at http://www.itu.int/ITU-R/.]

Liu, G., and Y. Fu, 2001: The characteristics of tropical precipitation profiles as inferred from satellite radar measurements. J. Meteor. Soc. Japan, 79, 131-143.

Lungu, T., 2001: QuikSCAT science data product user's manual, overview and geophysical data products. Version 2.2, Jet Propulsion Laboratory, California Institute of Technology Document JPL D-18053, 89 pp.

Mears, C. A., F. J. Wentz, and D. K. Smith, 2000: SeaWinds on QuikSCAT normalized objective function rain flag. Product description version 1.2, Remote Sensing Systems, 13 pp.

Naderi, F. M., M. H. Freilich, and D. G. Long, 1991: Spaceborne radar measurement of wind velocity over the ocean-An overview of the NSCAT scatterometer system. Proc. IEEE, 79, 850-866.

Petty, G. W., 1994: Physical retrievals of over-ocean rain rate from multichannel microwave imagery. Part 1: Theoretical characteristics of normalized polarization and scattering indices. Meteor. Atmos. Phys., 54, 77-99.

_ 2001: Physical and microwave radiative transfer properties of precipitating clouds. Part I: Principal component analysis of observed multichannel microwave radiances in tropical stratiform rainfall. J. Appl. Meteor., 40, 2105-2114.

Sauvageot, H., F. Mesnard, and R. S. Tenório, 1999: The relation between the area-average rain rate and the rain cell size distribution parameters. J. Atmos. Sci., 56, 57-70.

Spencer, R. W., 1986: A satellite passive $37 \mathrm{GHz}$ scattering-based method for measuring ocean rain rates. J. Climate Appl. Meteor., 25, 754-766.

— - H. M. Goodman, and R. E. Hood, 1989: Precipitation retrieval over land and ocean with the SSM/I: Identification and characteristics of the scattering signal. J. Atmos. Oceanic Technol., 6, 254-273.

Stamnes, K., S.-C. Tsay, W. Wiscombe, and K. Jayaweera, 1998: Numerically stable algorithm for discrete-ordinate-method radiative transfer in multiple scattering and emitting layered media. Appl. Opt., 27, 2502-2509.

Stiles, B. W., and S. H. Yueh, 2002: Impact of rain on spaceborne Ku-band wind scatterometer data. IEEE Trans. Geosci. Remote Sens., 40, 1973-1983.

Timothy, K. I., T. Iguchi, Y. Ohsaki, H. Horie, H. Hanado, and J. Kumagai, 1999: Test of the specific differential propagation phase shift (KDP) technique for rain-rate estimation with a Ku-band rain radar. J. Atmos. Oceanic Technol., 16, 10771091.

Tournadre, J., and Y. Quilfen, 2003: Impact of rain cell on scatterometer data: 1 . Theory and modeling. J. Geophys. Res., 108, 3225, doi:10.1029/2002JC001428.

Wentz, F. J., 1997: A well calibrated ocean algorithm for Special Sensor Microwave/Imager. J. Geophys. Res., 102, 8703-8718. , and R.W. Spencer, 1998: SSM/I rain retrievals within a unified all-weather ocean algorithm. J. Atmos. Sci., 55, 1613 1627.

, and D. K. Smith, 1999: A model function for the oceannormalized radar cross-section at $14 \mathrm{GHz}$ derived from NSCAT observations. J. Geophys. Res., 104, 11 499-11 514. 